\title{
Gastric Bleeding After Laparoscopic Spleen-Preserving Distal Pancreatectomy
}

\author{
Sven-Petter Haugvik, MD, Bård Ingvald Røsok, MD, PhD, Rune Andersen, MD, PhD, \\ Biørn Edwin, MD, PhD \\ Department of Hepato-Pancreato-Biliary Surgery, Rikshospitalet, Oslo University Hospital, Oslo, Norway \\ (Drs. Haugvik, Røsok, Edwin). \\ Institute of Clinical Medicine, University of Oslo, Oslo, Nonway (Drs. Haugvik, Edwin). \\ Department of Radiology and Nuclear Medicine, Rikshospitalet, Oslo University Hospital, Oslo, Norway (Dr. Andersen). \\ Interventional Centre, Rikshospitalet, Oslo University Hospital, Oslo, Norway (Dr. Edwin).
}

\begin{abstract}
Introduction: Laparoscopic spleen-preserving distal pancreatectomy for tumors of the pancreatic body and tail is becoming increasingly established at hepato-pancreato-biliary surgical departments worldwide. Spleen preservation is only recommended in benign or borderline lesions of the pancreas. We present a rare complication after laparoscopic spleenpreserving distal pancreatectomy.

Case Description: A 43-year-old woman with multiple endocrine neoplasia type 1 syndrome was referred to our department for surgical removal of a tumor in the pancreatic tail. A laparoscopic spleen-preserving distal pancreatectomy, including preservation of the splenic vessels, was performed. The patient was discharged on the tenth postoperative day after percutaneous drainage of peripancreatic fluid and transient fever. About 4 months postoperatively, she was admitted to her local hospital with recurrent anemia. Gastroscopy and abdominal computed tomography did not show any signs of bleeding, but prominent gastric varices and occlusion of the splenic vein were observed. The patient was referred back to our department, where an embolization of the splenic artery was performed with a percutaneous endovascular technique. She was discharged after 2 days with no recurrent anemia thereafter.
\end{abstract}

Discussion: Splenic artery embolization can be an effective treatment option for gastric variceal bleeding caused by splenic vein occlusion after laparoscopic spleen-preserving distal pancreatectomy.

Key Words: Laparoscopic pancreatic surgery, Distal pancreatectomy, Splenic artery embolization, Left-sided portal hypertension, Gastric variceal bleeding.

Citation Haugvik S-P, Røsok BI, Andersen R, Edwin B. Gastric bleeding after laparoscopic spleen-preserving distal pancreatectomy. CRSLS e2014.00306. DOI 10.4293/CRSLS.2014.00306.

Copyright (C) 2014 SLS This is an open-access article distributed under the terms of the Creative Commons Attribution-Noncommercial-ShareAlike 3.0 Unported license, which permits unrestricted noncommercial use, distribution, and reproduction in any medium, provided the original author and source are credited.

Address correspondence to: Sven-Petter Haugvik, MD, Department of Hepato-Pancreato-Biliary Surgery, Rikshospitalet, Oslo University Hospital, Sognsvannsveien 20, 0372 Oslo, Norway. Telephone: +47230747 02, Fax: +47 230725 26,

E-mail: svhaug@ous-hf.no

\section{INTRODUCTION}

Laparoscopic spleen-preserving distal pancreatectomy for tumors of the pancreatic body and tail is becoming increasingly established at hepato-pancreato-biliary surgical departments worldwide. The advantages of the minimally invasive approach are less intraoperative bleeding, ${ }^{1}$ faster postoperative recovery, ${ }^{2}$ shorter hospital stay, ${ }^{3}$ and improved cosmesis compared with the open approach. Spleen preservation is only recommended in benign or borderline lesions of the pancreas. There are 2 ways to preserve the spleen when one is performing a distal pancreatectomy-either by preserving the splenic vessels (splenic artery and vein) or by preserving the short gastric arteries and veins after division of the splenic vessels. The latter is known as the Warshaw technique. ${ }^{4}$

We present a rare complication after laparoscopic spleenpreserving distal pancreatectomy with preservation of the splenic vessels in a patient with multiple endocrine neoplasia type 1 syndrome. 


\section{CASE REPORT}

A 43-year-old woman with multiple endocrine neoplasia type 1 syndrome was referred to our department for surgical removal of a $28 \times 30-\mathrm{mm}$ tumor in the pancreatic tail. A laparoscopic spleen-preserving distal pancreatectomy with preservation of the splenic vessels was performed, after the surgical procedures described earlier. ${ }^{5}$ We used an endostapler (Endo GIA Black Reloads with Tri-Staple technology; Covidien, Dublin, Ireland) for transection of the pancreatic parenchyma and strengthened the staple line with a knotless wound closure device (V-Loc 180, size 3-0; Covidien).

On the second postoperative day, a fever developed and a significant elevation of the C-reactive protein level, from 74 to $313 \mathrm{~mL} / \mathrm{L}$, was registered. The serum amylase level was within the reference range ( $40 \mathrm{U} / \mathrm{L})$. An abdominal computed tomography (CT) scan performed on the same day showed diffuse fluid surrounding the pancreatic transection area and between the stomach and left liver lobe. No signs of gastric varices were described by the radiologist. The intraoperatively placed peripancreatic drain (Silisoft 19 Charriere; Péters Surgical, Bobigny, France) was removed because the CT scan showed suboptimal positioning of this drain. Ultrasonography-guided percutaneous drainage was planned but was not performed because the amount of fluid seen with ultrasonography did not allow for puncture. On the sixth postoperative day, ultrasonographic control of the peripancreatic fluid was performed. A $90 \times 60-\mathrm{mm}$ loculation between the stomach and left liver lobe was found, and with the patient under local anesthesia, a percutaneous $8 \mathrm{~F}$ pigtail catheter was introduced in the loculation. A thin yellowbrownish fluid was observed, but the amylase concentration of this fluid was not measured. On the next day, radiologic control with ultrasonography and abscessography with fluoroscopic spot filming did not show any remaining peripancreatic fluid, and the postoperatively placed drain was removed. The patient was discharged on the tenth postoperative day with a decreasing C-reactive protein level $(102 \mathrm{~mL} / \mathrm{L})$ and without pain.

About 1 month after surgery, the patient was admitted to her local hospital with melena and anemia, with a hemoglobin level of $4.7 \mathrm{~g} / \mathrm{dL}$. She received transfusion of red cells. Gastroscopy, colonoscopy, capsule endoscopy, and abdominal CT did not show any source of bleeding. She was discharged but was readmitted 3 months later with recurrent anemia. On the basis of new diagnostic data obtained from abdominal CT and gastroscopy, prominent gastric varices and an occlusion of the splenic vein were described for the first time (Figure 1).
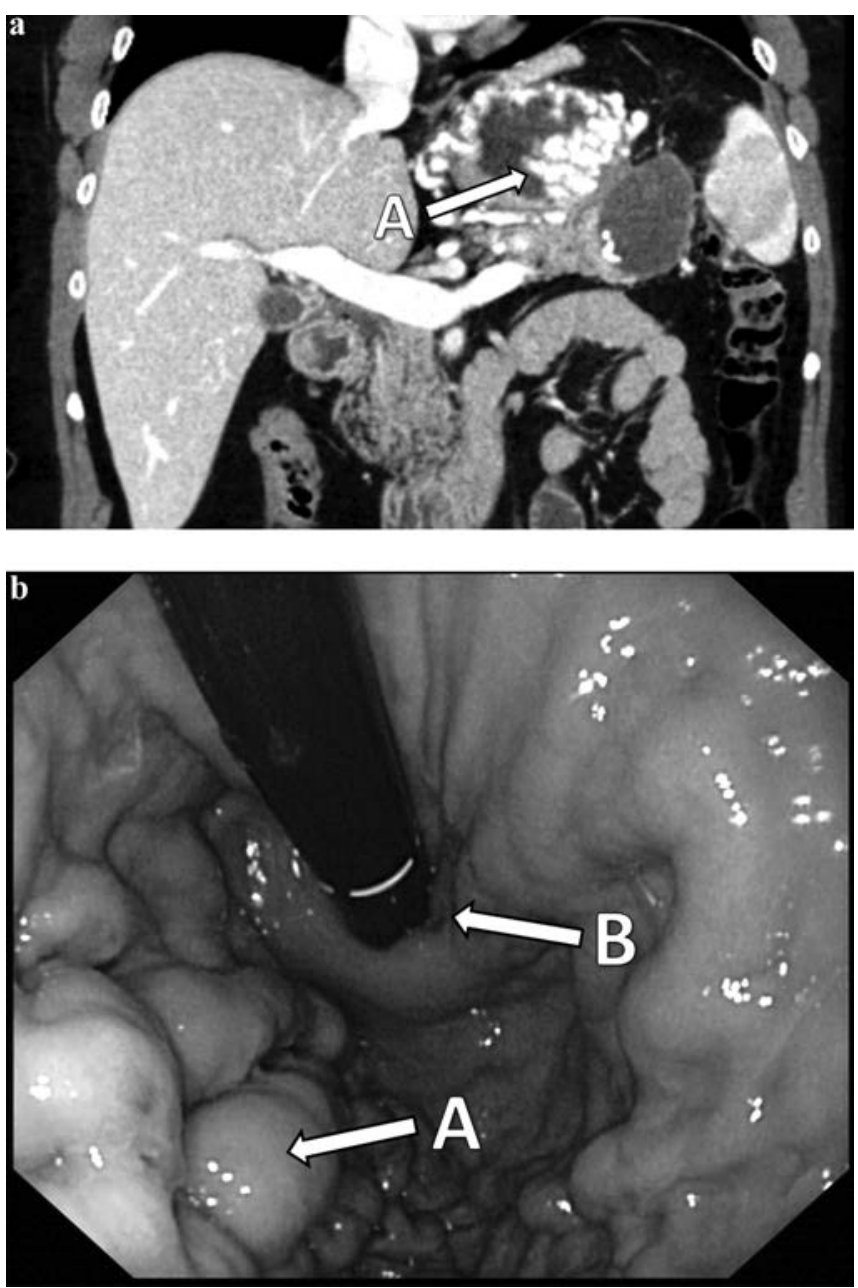

Figure 1. a, Abdominal CT scan before splenic artery embolization. (A, gastric varices.) b, Gastroscopy before splenic artery embolization. (A, gastric fundal varices; $\mathrm{B}$, endoscope at cardia.)

The patient was referred to undergo percutaneous transcatheter occlusion of the splenic artery in the interventional radiology suite (Figure 2). After standard catheterization of the right femoral artery, a 6F, 90-cm introducer tray (Check-Flo; Cook Medical, Bloomington, Indiana) was advanced to the proximal part of the splenic artery over a $6 \mathrm{~F}$ cobra guiding catheter, supported by a $4 \mathrm{~F}$ glide catheter and a 0.035 -inch glide wire (Terumo Interventional Systems, Tokyo, Japan). During fluoroscopic control, a vascular plug (Amplatzer Vascular Plug II; St. Jude Medical, Little Canada, Minnesota) with a diameter of 12 $\mathrm{mm}$ was placed proximal to the division of the splenic artery. The intra- and post-interventional courses were uneventful. The patient was discharged after 2 days, and at 17 months after intervention, she has not had any 

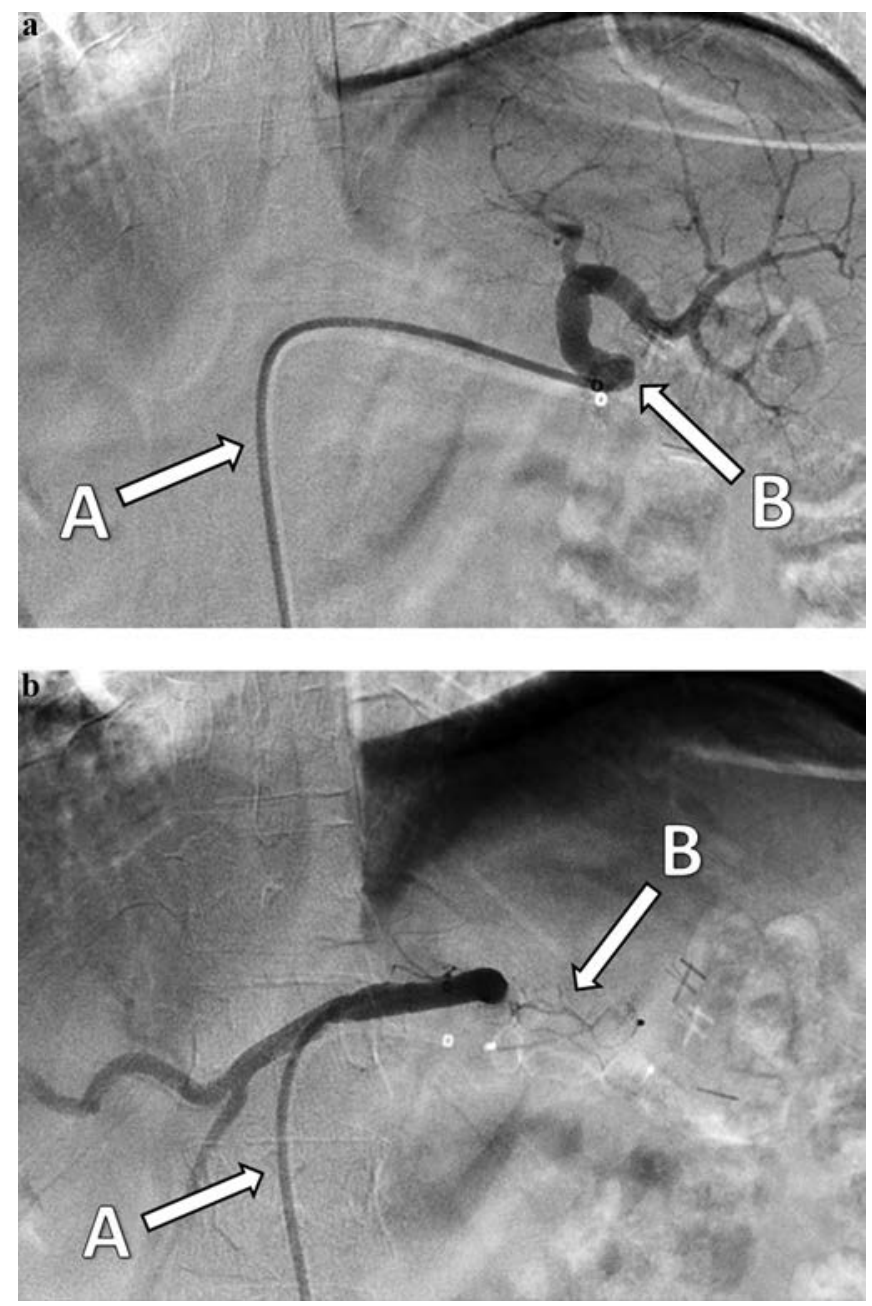

Figure 2. Digital subtraction angiography before and after splenic artery embolization. a, Angiography of splenic artery before embolization. (A, interventional catheter in splenic artery via right femoral artery; B, splenic artery.) b, Angiography of splenic artery after embolization. (A, interventional catheter in splenic artery via right femoral artery; B, vascular plug in splenic artery causing occlusion.)

recurrent anemia. Follow-up abdominal CT and gastroscopy did not show any gastric varices (Figure 3).

\section{DISCUSSION}

This case report is, to our knowledge, the first report of gastric variceal bleeding resulting from left-sided portal hypertension (LPH) due to occlusion of the splenic vein after spleen-preserving distal pancreatectomy with preservation of the splenic vessels.

LPH, also known as "sinistral" portal hypertension, is the result of an obstructed or thrombosed splenic vein that
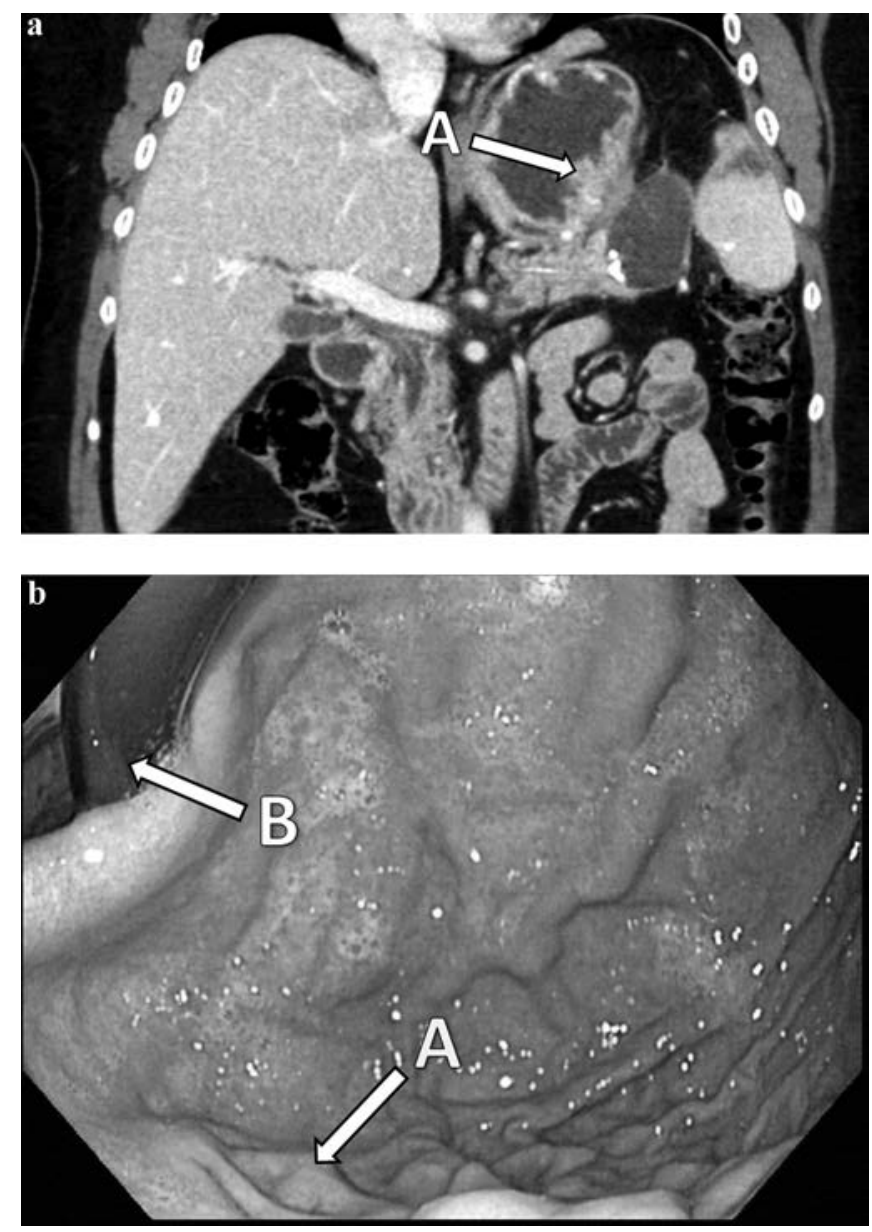

Figure 3. a, Abdominal CT scan after splenic artery embolization. (A, no gastric varices.) b, Gastroscopy after splenic artery embolization. (A, no gastric fundal varices; $\mathrm{B}$, endoscope at cardia.)

results in increased collateral circulation through the short gastric veins and left gastric vein and back into the portal system, with subsequent development of gastric varices. ${ }^{6}$ The pathophysiology of LPH was first described by Greenwald and Wasch ${ }^{7}$ in 1939. Most cases of LPH are the result of pancreatic diseases such as acute and chronic pancreatitis, pancreatic pseudocysts, or pancreatic carcinoma or pancreatic surgery with division of the splenic vessels. ${ }^{6,8,9}$ There are also nonpancreatic causes of $\mathrm{LPH}$, such as trauma, ${ }^{10}$ splenic cysts, ${ }^{11}$ and renal carcinoma. ${ }^{12}$ Gastric variceal bleeding caused by LPH is rare and occurs in about $4 \%$ of patients. ${ }^{13} \mathrm{LPH}$ should be suspected in patients with upper gastrointestinal bleeding and/or gastric varices, splenomegaly, and normal liver function. ${ }^{14}$

There are 2 curative treatment options for variceal bleeding caused by LPH-splenectomy ${ }^{15-18}$ and splenic artery embolization. ${ }^{19}$ Splenectomy is primarily indicated in patients with 
acute life-threatening hemorrhage. The advantages of splenectomy include prevention of hypersplenism and reduction of possible future recurrent bleeding, whereas the disadvantages include the risk of surgical morbidity or death and potential for overwhelming postsplenectomy infection. Splenic artery embolization has the advantage of being minimally invasive and preserving the immunologic function of the spleen. Thus prevention of surgical morbidity or death and overwhelming postsplenectomy infection is maintained. The disadvantages of splenic artery embolization include the risk of post-interventional splenic abscess and septicemia due to splenic infarction.

In the patient in this case report, obstruction of the splenic vein developed after laparoscopic spleen-preserving distal pancreatectomy with preservation of the splenic vessels. When the abdominal CT scan obtained on the second postoperative day was reassessed, the splenic vein was obstructed over a short distance near the pancreatic transection plane and the postoperatively developing peripancreatic fluid. This suggests that there may have been surgical reasons for the occurrence of LPH, such as thermal damage to the splenic vein through heat produced by the bipolar tissue sealing device applied or obstruction of the splenic vein associated with stapling of the pancreatic parenchyma, in our patient. On the basis of our experience with laparoscopic distal pancreatectomy, postoperative development of fluid in close relation to the pancreatic transection plane is relatively common without being associated with obstruction of the splenic vein. Thus it seems more probable that the venous obstruction was caused by technical surgical factors. However, the exact reason for the splenic vein occlusion remains unknown. Another observation that was made when the different postoperative CT scans were reassessed was a remaining and increasing loculation at the pancreatic transection plane, which seemed to obstruct the splenic vein increasingly over time. Simultaneously, gastric varices developed.

In conclusion, this report shows that splenic artery embolization can be an effective treatment option for gastric variceal bleeding caused by splenic vein occlusion after laparoscopic spleen-preserving distal pancreatectomy. The case presented illustrates a rare complication after pancreatic surgery that could have been diagnosed earlier, even though initial gastroscopy was without pathologic findings. When upper gastrointestinal bleeding is occurring after distal pancreatectomy, a 3-phase abdominal CT scan should be obtained in addition to gastroscopy to evaluate the possibility of LPH and associated gastric variceal bleeding. At our institution, we recommend curative treatment with emboliza- tion of the splenic artery in symptomatic patients with LPH without severe hemorrhage and recommend splenectomy in symptomatic patients with LPH and severe hemorrhage. The management of asymptomatic patients should be expectant, and these patients should be thoroughly informed about the potential risks of bleeding. ${ }^{20}$

\section{References:}

1. Limongelli P, Belli A, Russo G, et al. Laparoscopic and open surgical treatment of left-sided pancreatic lesions: clinical outcomes and cost-effectiveness analysis. Surg Endosc. 2012;26(7):1830-1836

2. Kim SC, Park KT, Hwang JW, et al. Comparative analysis of clinical outcomes for laparoscopic distal pancreatic resection and open distal pancreatic resection at a single institution. Surg Endosc. 2008;22(10):2261-2268.

3. Kooby DA, Hawkins WG, Schmidt CM, et al. A multicenter analysis of distal pancreatectomy for adenocarcinoma: is laparoscopic resection appropriate? J Am Coll Surg. 2010;210(5):779-777.

4. Warshaw AL. Conservation of the spleen with distal pancreatectomy. Arch Surg. 1988;123(5):550-553.

5. Rosok BI, Marangos IP, Kazaryan AM, et al. Single-centre experience of laparoscopic pancreatic surgery. Br J Surg. 2010; 97(6):902-909.

6. Sato T, Yamazaki K, Akaike J, Toyota J, Karino Y, Ohmura T. Clinical and endoscopic features of gastric varices secondary to splenic vein occlusion. Hepatol Res. 2008;38(11):1076-1082.

7. Greenwald HM, Wasch MG. The roentgenologic demonstration of esophageal varices as a diagnostic aid in chronic thrombosis of the splenic vein. J Pediatr. 1939;14:57-65.

8. Miura F, Takada T, Asano T, Ochiai T. Gastric varices occurring after middle-segment pancreatectomy preserving spleen with division of splenic artery and vein. Surgery. 2004;135(6):696-697.

9. Tien YW, Liu KL, Hu RH, Wang HP, Chang KJ, Lee PH. Risk of varices bleeding after spleen-preserving distal pancreatectomy with excision of splenic artery and vein. Ann Surg Oncol. 2010;17(8):2193-2198.

10. Bojal SA, Leung KF, Meshikhes AW. Traumatic pancreatic fistula with sinistral portal hypertension: Surgical management. World J Gastrointest Surg. 2010;2(7):251-254.

11. Kantarceken B, Cetinkaya A, Bulbuloglu E, Demirpolat G. Splenic hydatic cyst as a cause of sinistral portal hypertension and isolated gastric variceal bleeding. Turk J Gastroenterol. 2010; 21(3):317-320.

12. Joya S, del Valle LP, Marco MJ, Herrera MN, Agud Aparicio JL. Sinistral portal hypertension with bleeding gastric varices as 
initial manifestation of renal-cell carcinoma [in Spanish]. An Med Interna. 2004;21(6):283-284.

13. Heider TR, Azeem S, Galanko JA, Behrns KE. The natural history of pancreatitis-induced splenic vein thrombosis. Ann Surg. 2004;239(6):876-880.

14. Glynn MJ. Isolated splenic vein thrombosis. Arch Surg. 1986; 121(6):723-725.

15. Turrill FL, Mikkelsen WP. "Sinistral" (left-sided) extrahepatic portal hypertension. Arch Surg. 1969;99(3):365-368.

16. Kokabi N, Lee E, Echevarria C, Loh C, Kee S. Sinistral portal hypertension: presentation, radiological findings, and treatment options-a case report. J Radiol Case Rep. 2010; 4(10):14-20.
17. Jaroszewski DE, Schlinkert RT, Gray RJ. Laparoscopic splenectomy for the treatment of gastric varices secondary to sinistral portal hypertension. Surg Endosc. 2000;14(1):87.

18. Singhal D, Kakodkar R, Soin AS, Gupta S, Nundy S. Sinistral portal hypertension. A case report. JOP. 2006;7(6):670-673.

19. Gaba RC, Katz JR, Parvinian A, et al. Splenic artery embolization: a single center experience on the safety, efficacy, and clinical outcomes. Diagn Interv Radiol. 2013;19(1):49-55.

20. Loftus JP, Nagorney DM, Ilstrup D, Kunselman AR. Sinistral portal hypertension. Splenectomy or expectant management. Ann Surg. 1993;217(1):35-40. 